\title{
Foot Self-Care Behavior in Patients with Diabetes
}

\author{
Elif Turan ${ }^{1}$ Ramazan Önalan² ${ }^{2 \odot ~ N e c l a ~ C ̧ a y ~ I ̇ s ̧ c ̧ ı m e n ~}{ }^{2}$ \\ Yalçın Aral ${ }^{1 \oplus}$
${ }^{1}$ Depatment of Endocrinology, Faculty of Medicine, Yozgat Bozok University, Yozgat, Turkey
2Department of Internal Medicine, Faculty of Medicine, Yozgat
Bozok University, Yozgat, Turkey

\begin{abstract}
Address for correspondence Elif Turan, MD, Department of Endocrinology, Yozgat Bozok University, Adnan Menderes Street, 66040 Yozgat, Turkey (e-mail: drelifturan@hotmail.com).
\end{abstract}

J Soc Health Diab:2020;8:13-17

\begin{abstract}
Objective Diabetic foot ulcers (DFUs) are complications of diabetes that can progress with high mortality and morbidity. It is a preventable problem with the determination of risk factors and patient education. Our aim is to examine the knowledge and behavior of diabetic patients in our region about foot care.

Materials and Methods Diabetic individuals who applied to the Endocrine and Internal Medicine Clinic were included in this cross-sectional study. Demographic variables, physical examination, and laboratory findings were recorded. "Diabetic foot information form for diabetic foot care" was used to evaluate the patient's level of knowledge about diabetic foot and care. The questionnaire consists of 16 questions with two options, right or wrong, according to the suggestions of the guides. A total of 16 points scoring system has been established, 1 point for each correct answer and 0 points for the wrong answer. Results "Total acknowledgment scores" (TAS) were significantly higher in patients with DFU, amputation, and DFU history $(p<0.001)$. TAS were significantly lower in patients receiving oral antidiabetic therapy than those receiving insulin therapy $(p=0.005)$. Total scores were significantly lower in untrained patients compared with

Keywords

- diabetes

- diabetes foot

- education

- ulcer primary school graduates $(p=0.005)$, and in high school graduates compared with university graduates $(p<0.001)$.

Conclusion In this study, a serious lack of information or insufficiency was found in patients with diabetes. More comprehensive training programs are needed to reduce diabetic foot development and complications.
\end{abstract}

\section{Introduction}

Diabetes mellitus (DM) is a metabolic disease with acute and chronic complications. Diabetic foot ulcers (DFUs), on the other hand, are one of the complications in which diabetes patients are affected by approximately $25 \%$ of their lives. ${ }^{1}$ Diabetic foot (DF) is the most common reason for hospitalization in patients with diabetes, and its mortality and morbidity are high. ${ }^{2,3}$ Quality of life and social activities of patients with DF are severely affected because of pain, inactivity, and loss of sensation. The first reason for foot amputations is known as DF, and foot amputation rate in diabetic patients is $24 \%{ }^{4}$

DOI https://doi.org/ $10.1055 / \mathrm{s}-0040-1716812$ ISSN 2321-0656.
DFU develops due to changes in foot pressure foci due to diabetic neuropathy, blood loss in diabetic vasculopathy, and recurrent trauma. In unhealed DFUs likely to go to amputation, the first goal is to protect the patient from lower limb amputations. ${ }^{5}$ According to the American Diabetes Association, early diagnosis and treatment of diabetic patients can prevent ulcers and amputation or other negative consequences of risky feet. ${ }^{6}$ For this purpose, it is necessary to recognize the feet at risk, to take effective treatment and measures. Education is the most important part of success in prevention. Some studies have shown that regular foot self-care significantly reduces lower limb amputations. ${ }^{\text {? }}$

This is an open access article published by Thieme under the terms of the Creative Commons Attribution-NonDerivative-NonCommercial-License, permitting copying and reproduction so long as the original work is given appropriate credit. Contents may not be used for commercial purposes, or adapted, remixed, transformed or built upon. (https://creativecommons.org/licenses/by-nc-nd/4.0/).

Thieme Medical and Scientific Publishers Pvt. Ltd. A-12, 2nd Floor, Sector 2, Noida-201301 UP, India 
Turkey Diabetes, Hypertension, Obesity and Endocrinology Diseases Prevalence Study (TURDEP-II) showed an alarming increase in diabetes rates in Turkey. According to the study, the frequency of diabetes has been reported to have increased by $90 \%$ over the past 12 years from 7.7 to $13.7 \%{ }^{8}$ In parallel with this increase, diabetes complications are likely to increase. Therefore, it is very important to prevent complications such as DF, which brings serious social and economic problems. In this study, it was aimed to evaluate the awareness and behavior of DF care in our region.

\section{Materials and Methods}

In this cross-sectional study, outpatients and inpatients admitted to the Endocrine and Internal Medicine Clinics of our hospital between September 2018 and June 2019 were included. Age, gender, height, weight, body mass index $\left(\mathrm{kg} / \mathrm{m}^{2}\right)$, type of diabetes, duration of diabetes, drugs, history of smoking, and history of DF problems were recorded. On physical examination, blood pressure, peripheral pulses, and neurological examination were evaluated. Laboratory findings of glucose, hemoglobin A1c (HbA1c), lipid profile, and other biochemical and hematological parameters were noted.

"The diabetic foot information form for diabetic foot care" was used to assess the patient's level of acknowledge about DF and care. In our country, there is no valid and reliable measurement tool to measure the level of knowledge. Therefore, a questionnaire was prepared according to the principles of DF care and prevention. The questionnaire consists of 16 questions with two options as right or wrong according to the recommendations of the IDF Clinical Practice Recommendations on the Diabetic Foot 2017, ${ }^{9}$ Preventive Foot Care. ${ }^{10,7} \mathrm{~A}$ total of 16-point scoring system was created, 1 point for each correct answer and 0 points for the wrong answer (-Table 1$)$. Each patient was given diabetes education after the questionnaire.

The study protocol was in accordance with the Helsinki Declaration of Human Rights and was confirmed by the Yozgat Bozok University Clinical Research Ethics Committee in August 13, 2018 (2017-KAEK-189_2018.09.12_02) and informed consent form was taken from all participants.

\section{Statistical Analyses}

All statistical analyses were calculated with the Statistical Package for the Social Sciences, version 15.0 (Chicago, Illinois, United States). A $p$-value of $<0.05$ was considered as statistically significant. The characteristics of participants were compared using an analysis of variance and Student's $t$-test for normal distributions. $t$-Test was used in normal distributions and Mann-Whitney $U$ and Kruskal-Wallis were used in the abnormal distribution. Stepwise linear regression analysis was performed to determine the interfering factors from among the patients' demographics with the assumption of the total knowledge score and the total score after education as the dependent variables, respectively.

\section{Results}

Three hundred and fifty-four diabetic patients were included in the study. The mean age of the participants was $59.44 \pm 11.85$ years. Note that $61.3 \%$ (217) were females and $38.7 \%$ (137) males. Note that $2.3 \%$ of them had type 1 diabetes. The mean diabetes duration of patients was $9.64 \pm$ 7.27 years. Eighty-three percent of participants had smoking

Table 1 The responses to questions related to the knowledge of foot care

\begin{tabular}{|l|l|l|}
\hline Questions related to knowledge of foot care & Correct (\%) & Wrong (\%) \\
\hline $\begin{array}{l}\text { Diabetes patients should take regular medication to prevent complications related to diabe- } \\
\text { tes (kidney, nerve, heart, eye problems) }\end{array}$ & 85.6 & 14.4 \\
\hline Diabetes patients should check their feet as they cannot feel minor traumas & 35.6 & 64.4 \\
\hline In diabetes patients, infection and wounds should not be easily healed should check their feet & 37 & 63 \\
\hline They should check their feet as they may have foot wounds & 39.5 & 60.5 \\
\hline Diabetes patients should not smoke because smoking can affect blood circulation & 46.3 & 53.7 \\
\hline Diabetes patients should examine the feet every day & 9.3 & 91.7 \\
\hline If you see redness, bleeding between the nails, you show the doctor & 42.2 & 57.8 \\
\hline Do you do nail cutting weekly? & 87 & 35.6 \\
\hline Do you wear comfortable shoes that do not squeeze? & 64.4 & 21.5 \\
\hline Do you wash your feet ever day? & 78.5 & 72.3 \\
\hline If you wash your feet, will you dry your fingers? & 27.7 & 87 \\
\hline Do you always check the temperature of the water before putting your feet in the water? & 13 & 63 \\
\hline Do you wash your feet with warm water? & 37 & 95.2 \\
\hline Can you check the soles of your shoes for any foreign matter or lining in every clothing? & 4.8 & 65.8 \\
\hline Can you put a moisturizer on the soles? & 34.2 & 29.9 \\
\hline Can you walk barefoot? & 70.1 & \\
\hline
\end{tabular}

Note: Wrong: false and do not know. 
habit. The demographic features are shown in - Table 2 and diabetic complications rate in - Table 3 .

Of the 354 patients, 84 (23\%) had no formal education, 212 (59.8\%) had primary education, 34 (9,6\%) graduated high school and 24 (6.7\%) graduated from university.

Note that $10.2 \%$ (36) of patients had a history of foot ulcers. A total of $2.5 \%$ (9) of the patients had previous foot, toe, or leg amputation. Note that $8.2 \%$ of patients had sores, ulcers, or blisters on the foot. Thirteen percent (46) of patients had callus on their feet. Twenty-six percent (92) of the patients had diabetic neuropathy.

\section{Knowledge of Foot Care}

The mean total acknowledge score (TAS) was $6.84 \pm 3.53$. Note that $9.3 \%$ (33) of them check their feet every day. Note that $4.8 \%$ (17) of diabetic patients check the soles of their shoes for any foreign matter or lining in every clothing. - Table 4 shows the factors affecting the TAS. TAS were significantly higher in patients with DFU, amputation, and DFU history $(p<0.001)$. The TAS was significantly lower in patients receiving oral antidiabetic therapy than insulin treatment $(p=0.005)$.

Table 2 Demographic features of all participants $(n=354)$

\begin{tabular}{|c|c|}
\hline Parameter & $\begin{array}{l}\text { Mean } \pm \text { standard deviation } \\
\text { Median } \\
\text { (minimum-maximum) }\end{array}$ \\
\hline Gender (female/male) & $217 / 137$ \\
\hline Age (y) & $59.44 \pm 11.85$ \\
\hline Height (cm) & $164.86 \pm 8.20$ \\
\hline Weight $(\mathrm{kg})$ & $84.0 \pm 13.67$ \\
\hline Body mass index $\left(\mathrm{kg} / \mathrm{m}^{2}\right)$ & $30.50 \pm 5.44$ \\
\hline Diabetic duration (year) & $9.64 \pm 7.27$ \\
\hline Fasting glucose (mg/dL) & $163.11(62-440)$ \\
\hline Total cholesterol (mg/dL) & $193.81 \pm 45.09$ \\
\hline $\mathrm{HDL}(\mathrm{mg} / \mathrm{dL})$ & $47.40 \pm 12.70$ \\
\hline LDL (mg/dL) & $111.17 \pm 38.81$ \\
\hline Triglyceride (mg/dL) & $175.58(35-932)$ \\
\hline HbA1c (\%) & $7.88(5.7-16)$ \\
\hline Leucocyte, $10^{3} / \mu \mathrm{L}$ & $8.66 \pm 2.46$ \\
\hline $\mathrm{Hb}, \mathrm{g} / \mathrm{dL}$ & $13.70 \pm 1.80$ \\
\hline Platelet, $10^{3} / \mu \mathrm{L}$ & $287.09 \pm 78.86$ \\
\hline Mean systolic pressure $(\mathrm{mm} \mathrm{Hg})$ & $140.89 \pm 20.04$ \\
\hline $\begin{array}{l}\text { Mean diastolic } \\
\text { pressure }(\mathrm{mm} \mathrm{Hg})\end{array}$ & $88.41 \pm 11.34$ \\
\hline
\end{tabular}

Abbreviations: Hb, hemoglobin; HbA1c, hemoglobin A1c; HDL, high-density lipoprotein; LDL, low-density lipoprotein.

Table 3 Diabetic complications rate

\begin{tabular}{|l|l|l|}
\hline Complications $(\boldsymbol{n}=\mathbf{3 5 4})$ & Absent (\%) & Present (\%) \\
\hline Diabetic neuropathy & 73.7 & 26.3 \\
\hline Diabetic nephropathy & 61.2 & 39.8 \\
\hline Peripheral artery disease & 73.9 & 26.1 \\
\hline
\end{tabular}

The TAS were $4.83 \pm 2.81$ in patients with no formal education, $7.22 \pm 3.49$ in patients with primary education, $7.36 \pm 2.66$ in patients that graduated high school, and $9.71 \pm 3.79$ in patients that graduated university. Total scores were significantly lower in patients with no formal education compared with primary school graduates $(p=0.005)$, and in high school graduates compared with university graduates $(p<0.001)$ (-Fig. 1).

In correlation analysis, TAS was negatively correlated with age $(r=-0.18 p=0.01)$, and positively correlated with duration of diabetes $(r=0.136, p=0.01)$, glucose $(r=0.117$, $p=0.015)$, and $\operatorname{HbA1c}(r=0.13, p=0.015)$.

We analyzed linear regression to investigate the interference of each of the variables on the TAS of the diabetics. Age (confidence interval [CI]: 0.07-0.062, $p=0.015$ ), diabetes foot education (CI: 3.53-5.37, $p<0.001$ ), patient education level (CI: $0.73-1.52, p<0.001$ ), and history of DFU (CI: 2.03-4.02, $p<0.001$ ) were found to be independent predictors of the total score.

\section{Discussion}

Our study demonstrated that the majority of patients with diabetes do not have sufficient knowledge and skills in foot care. Inexperience in nail care, foot hygiene, and controls, interpretation of DFU symptoms (redness), effects of smoking on DFU formation, and selection of suitable shoes were determined.

Education is directly related to DF care. Knowledge and self-care were found seriously insufficient in individuals with low education level. The scores of diabetic individuals with low education level were found to be quite low. The relationship between education and information can also be explained by the fact that the educated patient can use educational materials and information technologies to obtain information. Reports from India, Iran, and Pakistan also support these results. ${ }^{11-13}$

Studies to prevent DF wounds emphasizes the importance of blood sugar regulation, proper footwear, foot and nail care, regular foot examinations, and patient education. ${ }^{14,15}$ The purpose of DM training programs with including the patient in the management of the disease is to improve metabolic control, to prevent acute and chronic complications, and to improve the quality of life. Only 42 (13.4\%) patients received diabetes and DF training.

People with diabetes are likely to have 15 to 40 times more knee-leg amputations than the general population. The presence of DFU is 36 times more likely to lead to future ulcer development. ${ }^{9}$ In our study, TAS was found to be significantly higher in patients with a history of DFUs and amputation.

Diabetic peripheral neuropathy (DPN) is one of the main factors of DFU. In a multicenter study conducted, coexistence of minor trauma, deformity, and DPN has been shown to account for $63 \%$ of DFU. ${ }^{16}$ Due to diabetic neuropathy, the pressure foci of the feet change, and the feeling of pain decreases, and the feet become vulnerable. Shoes that do not match should not be worn, the method used to warm the 
Table 4 Factors on knowledge of foot care

\begin{tabular}{|l|l|l|}
\hline Factors (number) & Mean \pm standard deviation & p-Value \\
\hline Female $(n=217)$ & $6.43 \pm 3.44$ & 0.86 \\
Male $(n=137)$ & $7.50 \pm 3.56$ & $<\mathbf{0 . 0 0 1}$ \\
\hline History of DFU, Yes $(n=47)$ & $9.64 \pm 3.66$ & $<.42 \pm 3.304$ \\
No $(n=307)$ & $12.33 \pm 3.55$ & $\mathbf{0 . 0 0 1}$ \\
\hline History of amputation due to (DFU), Yes $(n=9)$ & $6.70 \pm 3.44$ & $\mathbf{0 . 0 0 1}$ \\
No $(n=345)$ & $10.14 \pm 3.47$ & 0.735 \\
\hline DFU, Yes $(n=29)$ & $6.54 \pm 3.38$ & \\
No $(n=325)$ & $7.02 \pm 3.64$ & 0.064 \\
\hline Callus on feet, Yes $(n=46)$ & $6.83 \pm 3.50$ & \\
No $(n=308)$ & $7.43 \pm 3.67$ & $\mathbf{0 . 0 0 5}$ \\
\hline Diabetic neuropathy, Yes $(n=92)$ & $6.64 \pm 3.45$ & \\
No $(n=162)$ & $7.80 \pm 3.81$ & $<.24 \pm 3.19$ \\
\hline Insulin treatment, Yes $(n=137)$ & $12.09 \pm 2.13$ & $\mathbf{0 . 0 0 1}$ \\
No $(n=217)$ & $6.46 \pm 3.29$ & \\
\hline Diabetic foot education, Yes $(n=42)$ & & \\
No $(n=313)$ & & \\
\hline
\end{tabular}

Abbreviation: DFU, diabetic foot ulcer.

Note: Values of $p<0.05$ were considered as statistically significant.

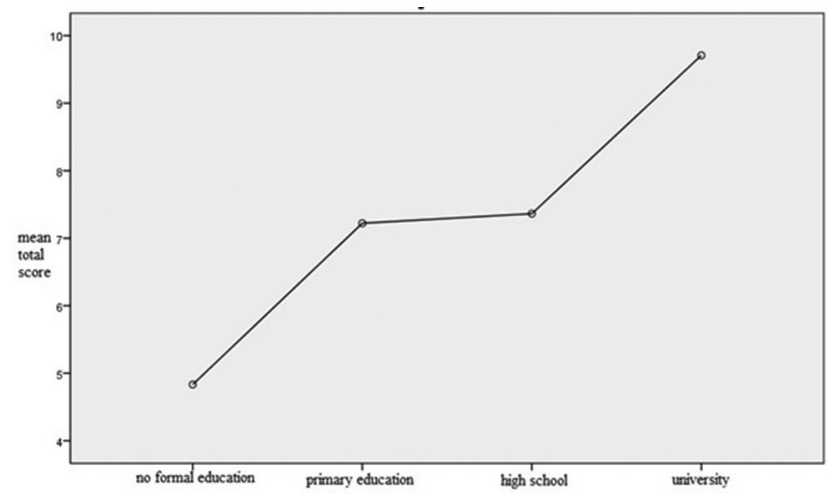

Fig. 1 Relationship between total acknowledgment score and level of education.

cold feet and trainings on nonbarefoot gain more importance for patients with diabetic neuropathy. In our study, no significant difference was found in TAS compared with those without neuropathy. In addition, there was no significant difference in TAS in patients with callus due to varying foci of pressure on the foot. This situation was interpreted as a reflection of the lack of education observed throughout the study.

The results of the studies conducted with the DF knowledge level differ according to the regions. While the level of knowledge was low in one study in Sudan, the level of knowledge was found good in another study in Saudi Arabia. ${ }^{17,18}$ This difference may be related to the participants living in urban and rural areas, accessing health centers, and the number of health care professionals in the region. In a study conducted in China, low self-care skills were associated with education, duration of diabetes, lack of regular periodic examinations, and diabetes complication training. ${ }^{19}$ It can be said that regular doctor control increases awareness of foot care. ${ }^{20}$
There are some limitations of this study. First, the knowledge level form does not include all of the knowledge and practices related to DF care. Second, the questionnaire we used was not confirmed by the test-retest method. To minimize the margin of error, locally and internationally accepted rules were used to prepare the survey. ${ }^{21-23}$ Finally, although a sufficient number of patients participated in the study, as it is a single-center study it does not represent Turkey's profile.

\section{Conclusion}

As a result, diabetes and DF education of the patients were poor and this was closely related to the patient's educational status and DF education. Although diabetes and DF education progress with special effort of the clinics, it does not seem sufficient. Patients need more information about diabetes and DF by health care professionals.

\section{Funding}

None.

\section{Conflict of Interest}

None declared.

\section{References}

1 Turan E, Diyabetik ayak epidemiyolojisi ve risk faktörleri. In: Turan E, ed. Diyabetik ayak tanı ve tedavisinde multidisipline yaklaşım. Ankara: Dünya Tıp Kitabevi; 2019 19-26

2 Mason J, O'Keeffe C, Hutchinson A, McIntosh A, Young R, Booth A. A systematic review of foot ulcer in patients with type 2 diabetes mellitus. II: treatment. Diabet Med 1999;16(11):889-909

3 Lim JZM, Ng NSL, Thomas C. Prevention and treatment of diabetic foot ulcers. J R Soc Med 2017;110(3):104-109

4 Vatankhah N, Khamseh ME, Noudeh YJ, Aghili R, Baradaran HR, Haeri NS. The effectiveness of foot care 
education on people with type 2 diabetes in Tehran, Iran. Prim Care Diabetes 2009;3(2):73-77

5 Armstrong DG, Boulton AJM, Bus SA. Diabetic foot ulcers and their recurrence. N Engl J Med 2017;376(24):2367-2375

6 Association AD; American Diabetes Association. 11. Microvascular complications and foot care: standards of medical care in diabetes-2019. Diabetes Care 2019;42(Suppl 1):S124-S138

7 Everett E, Mathioudakis N. Update on management of diabetic foot ulcers. Ann N Y Acad Sci 2018;1411(1):153-165

8 Satman I, Omer B, Tutuncu Y, et al; TURDEP-II Study Group. Twelve-year trends in the prevalence and risk factors of diabetes and prediabetes in Turkish adults. Eur J Epidemiol 2013;28(2): 169-180

9 Ibrahim A. IDF Clinical Practice Recommendation on the Diabetic Foot: a guide for healthcare professionals. Diabetes Res Clin Pract 2017;127:285-287

10 Mayfield JA, Reiber GE, Sanders LJ, Janisse D, Pogach LM; American Diabetes Association. Preventive foot care in diabetes. Diabetes Care 2004;27(Suppl 1) :S63-S64

11 Khamseh ME, Vatankhah N, Baradaran HR. Knowledge and practice of foot care in Iranian people with type 2 diabetes. Int Wound J 2007;4(4):298-302

12 Hasnain S, Sheikh NH. Knowledge and practices regarding foot care in diabetic patients visiting diabetic clinic in Jinnah Hospital, Lahore. J Pak Med Assoc 2009;59(10):687-690

13 Viswanathan V, Shobhana R, Snehalatha C, Seena R, Ramachandran A. Need for education on footcare in diabetic patients in India. J Assoc Physicians India 1999;47(11): 1083-1085

14 Ragnarson Tennvall G, Apelqvist J. Health-economic consequences of diabetic foot lesions. Clin Infect Dis 2004; 39(2, Suppl 2) :S132-S139

15 Younes NA, Albsoul AM, Awad H. Diabetic heel ulcers: a major risk factor for lower extremity amputation. Ostomy Wound Manage 2004;50(6):50-60
16 Boulton AJ, Vileikyte L, Ragnarson-Tennvall G, Apelqvist J. The global burden of diabetic foot disease. Lancet 2005;366(9498) : 1719-1724

17 Gumaa MM, Shwaib H, Ali S. Diabetic foot lesions predicting factors, view from Jabir Abu-alaiz diabetic centre in Khartoum, Sudan. J Diab Foot Complications 2016;8:6-17

18 Algshanen MA, Almuhanna MF, Almuhanna AM, et al. Diabetic foot awareness among diabetic patients in Saudi Arabia. Egypt J Hosp Med 2017;68(2):1289-1290

19 Li R, Yuan L, Guo X-H, et al. The current status of foot self-care knowledge, behaviours, and analysis of influencing factors in patients with type 2 diabetes mellitus in China. Int J Nurs Sci 2014;1(3):266-271

20 Goie TT, Naidoo M. Awareness of diabetic foot disease amongst patients with type 2 diabetes mellitus attending the chronic outpatients department at a regional hospital in Durban, South Africa. Afr J Prim Health Care Fam Med 2016;8(1):e1-e8

21 Pinzur MS, Slovenkai MP, Trepman E; The Diabetes Committee of the American Orthopaedic Foot and Ankle Society. Guidelines for diabetic foot care. Foot Ankle Int 1999;20(11):695-702

22 Bakker K, Apelqvist J, Schaper NC; International Working Group on Diabetic Foot Editorial Board. Practical guidelines on the management and prevention of the diabetic foot 2011 Diabetes Metab Res Rev 2012;28(Suppl 1) :225-231

23 Newrick P, International consensus on the diabetic foot. BM] 2000;321(7261):642A 